\title{
Review Paper: Technologies for Facial Emotion Recognition and Chatbots for Depression Handling
}

\author{
Mayuri Solase \\ Student \\ Dr. Dy Patil School of Engineering \\ Academy, Ambi, \\ Pune, India
}

\author{
Sonam Pedgaonkar \\ Student \\ Dr. Dy Patil School of engineering \\ academy, Ambi, \\ Pune, India
}

\author{
Mayuresh Pathade \\ Student \\ Dr. Dy Patil School of Engineering \\ Academy, Ambi, \\ Pune, India
}

\begin{abstract}
With the improvement in technology, people are getting nearer through internet society but facing more challenges in their daily life and their interaction with other people. Less interaction can cause one to feel depressed and since people are still busy maintaining their privacy, they are not able to talk about it. In that case, they have to find a way to maintain their mental health in their closest technology. This paper reviews available technologies that can detect facial expressions and how they can be used for mental health. This paper also includes a survey on how the Dialogflow framework can be used to implement a chatbot and help to improve mental health. The main issue that came in this review was to merge the facial recognition and the chatbot part of the app. This issue can be solved by using the IONIC framework since facial recognition and Dialogflow can be embedded in ionic.
\end{abstract}

\section{General Terms}

Facial emotion recognition APIs, Mental health, Depression handling chatbot.

\section{Keywords}

FaceApi, Dialogflow, api.ai, face expression detection APIs, Ionic framework.

\section{INTRODUCTION}

From the start of the 20th century, technology is advancing vastly. The improvements in technology are creating life much easier but it is also creating a big impact on people. People are feeling freer since the internet and other entertaining things have come. But on the other side, the meter of depression is getting higher and higher in daily life. And nowadays, it has become more difficult to express and deal with those negative emotions. In this technological era, smartphones can help people to detect their depression level using some algorithms and notify them [26]. This paper tries to create an app that will help the user to overcome their depression by suggesting to them the ways to tackle it. The app is made using the Ionic 4 framework and has a feature that will detect the user's emotions using their face. Here an open-source face-emotion detector can be used inside the ionic app. For the handling of all other parts after the detection, the Dialogflow framework can also be a good choice. The main challenge will be to combine these 3 independent technologies and create an app that will help the user to overcome their depression.

\section{DISCUSSION}

\subsection{Mental Health Using Mobile Apps}

Depression is a common and serious illness that affects human thinking and behavior. But it is curable. Depression causes one to feel sad and lose their interest in some things which becomes a reason to decrease in working abilities of a person [1]. In this era of technology, people are still hesitating to talk about it. Only a few of them go to the doctor but a large number of people are not comfortable to talk about it. In this technological era, one can use technology to help them feel less depressed. New technology can find whether one is depressed or not and can help them to elevate their depression [1]. Some applications are available and can help people to fight their depression. There are various apps like WoeBot, Wysa, Tess, Youper[25] which help the user to administer their mental health. But they cannot detect depression they can only treat it [2].

\subsection{Depression Detection in Apps}

The main thing is that the detection of depression is difficult and it cannot be easily done. There are many ways by which depression can be detected like handwriting, chats, twitter feed analysis, voice analysis, and visual cues. Detection of depression should be done using the things which are highly related to human behavior, like voice and facial expressions [3]. Vinod Bharat presented some data mining techniques for this purpose [31]. Nilesh Wani and LS Malphedwar also proposed an image processing technique [30] for web-based images. Since many apps are based on languages like android, angular, etc. The depression detection cannot be done using languages like python. So, the best way is to make an app that can detect depression using basic languages like android, angular. Face emotion recognition in apps can be used which can recognize the user's expression and act as per their expressions. [6]. There are some platforms for face recognition provided by Google and Microsoft.

\subsection{Microsoft vs Google face detection}

Many Cloud-based APIs are available for facial emotion recognition and detection. Microsoft and Google have their APIs for this case. Microsoft's Azure and Google's API were compared by Salik Ram Khanal and team [10] using a dataset of 980 images with many poses of the face.Both API had to decide which of the 8 emotions (Anger, Afraid, Disgust, Happiness, Neutral, Sadness, Surprise) the image contain. The results were that Microsoft Azure was better in straight face images but face detection was better in Google. Microsoft has a true-positive value of up to $60 \%$ and google has $45.25 \%$. [10].

\subsection{FaceAPI for Facial Expression}

Vincent Muhler created an API called faceapi.js which can detect a face, recognize the face and detect face emotions. It uses tensorflow.js as a base and implements the technology. This technology proposed a facial recognition API which is based on tensorflow.js and uses it to help the user to detect face and emotions. It can also detect face and emotions from live video. 
The structure of faceapi can be like this.

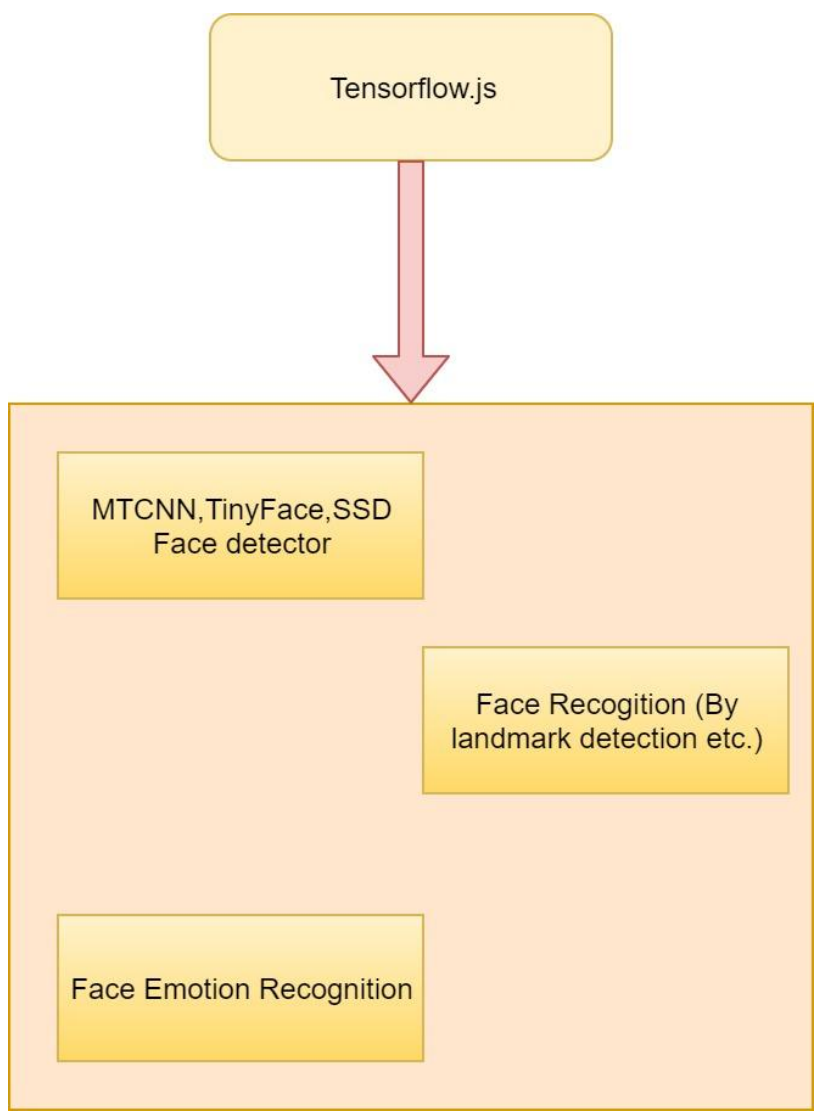

Fig.1 FaceApi Structure

\subsection{Handling depression using Ai}

Detection of depression is only one step to handle the depression after that, the focus should be to handle the depressed person more carefully. Now it is the era of Artificial Intelligent Assistants.

And with the help of that, it is easier to create a chatbot which will help the depressed person to fight their depression. It is possible to implement a chatbot in various ways. There are many ways to implement a chatbot and there are many organizations that are researching AI assistants to help the depressed. Some chatbots can help to elevate depression [5]. The main thing is to create a chatbot and use it in the app. Right now, various platforms help to create our chatbots and customize them. Google's Dialogflow is also the best option to create a chatbot. DINA a chatbot used to manage the university admission process [7] and some chatbots that can help railway systems to handle user queries and answer them [8] was built using this technique. There are also some ontology-based chatbots like Eliza which can simulate a therapist [6]. Dialogflow helps to build an AI which can be embedded in the app and used.

By using new technologies and programming languages or frameworks developing the new code for AI behavior in Dialogflow can be done which avoids similarities in the behavior of the same platform chatbots.

\section{CHALLENGES TO IMPLEMENT CHATBOTS}

The main problem is also that face detection is not precise; its accuracy is still around $70-80 \%$ and needs very particular face reactions to distinguish them. There are some people whose facial expressions are not so powerful. In this case, face emotion detection fails and this issue is still unsolved.

There are also some challenges in building chatbots [19] which are selection type of chatbots and as well as the programming language of chatbots.

Another challenge is to handle and merge these two technologies. Vincent Muhler's faceapi is based on Angularlike programming [4]. And Dialogflow provides support to many major languages. Hence a platform that can merge them together is a must.

One solution to this problem is the IONIC framework. Using the ionic framework an app that is compatible with both Android and IOs platforms can be developed. IONIC has an MVC framework and uses component schema which can be used to add Dialogflow chatbot as a service to the application. And with the help of this, it is easier to implement the facial recognition technology in ionic due to its angular support. It is also helpful to deploy the app and make its versions.

Right now, training set is also a big issue in recognizing intent through chatbots. [11]

\section{CONCLUSION}

From all the above discussions the conclusion is that the detection of the depression and provide the solution to it is not that easy task. Microsoft and Google have their APIs for this case. But both the Api was doing two different things as Microsoft was better in image processing of different emotions and Google was better in emotion detection. But both of it was not that much of impactful to recover the depressed person.

Since there is the solution on it which is that the depression can be detected using the FaceApi which uses the TensorFlow framework as its base. Face Expression can be detected using these APIs and then using the Dialogflow the app can be connected to the AI.

\section{FUTURE SCOPE}

By using the various technologies, it gets this new approach to create applications using the Ionic framework which may reduce the coding and designing part in the future. The Ionic app can be easily enhanced using any other functionality and their APIs to provide good AI support.

Using this framework, it is possible to implement an app that can help to elevate depression. The apps that can detect depression and as well as help users like a real professional can be developed.

If the emotion detection accuracy increases and it can detect emotions from small changes in faces it will be a big advantage in facial emotion recognition systems.

\section{REFERENCES}

[1] Lorny P 2019, 4 ways to tackle depression. .

[2] Jodi Clarke 2019, Can artificial intelligence help with depression?

[3] Anastasia Pampouchidou, Kostas Marias and Fan yang, 2017, Automatic assessment of Depression Based on Visual Cues.

[4] Vincent muhler 2018, ITNEXT, faceiapi.js- JavaScript API for Face Recognition in Browser with tensorflow.js.

[5] Gillian Cameron, David Cameron, Gavin Megaw, 2017 Towards a chatbot for digital counselling. 
[6] AnushaVegesna, Pranjal Jain, DhruvPorwal, 2018, Ontology based Chatbot (For E-commerce Website)

[7] Heru Agus Santoso, Galuh Wilujeng Saraswati, Muhammad Syaifur Rohman, 2018. Dinus Intelligent Assistance (DINA) Chatbot for University Admission Services.

[8] Satyanarayana, Rajesh Budihal, 2019. Chatbot for Railway using DilougFlow.

[9] Mandar Deshpande, Vignesh Rao 2017. Depression Detection using Emotion Artificial Intelligence.

[10] Salik Ram Khanal, Vítor Filipe, Jaime Sampaio , Nuno Lopes, 2017, Performance analysis of Microsoft's and Google's Emotion Recognition API using pose-invariant faces.

[11] Lars Bollweg, Maik Kurzke, Asif Shahriar, Peter Weber, 2018, When Robots Talk - Improving the Scalability of Practical Assignments in MOOCs Using Chatbots.

[12] Xiuzhuang Zhou, Kai Jin, Yuanyuan Shang, and Guodong Guo, 2018, Visually Interpretable Representation Learning for Depression Recognition from Facial Images.

[13] Sarmad Al-gawwam, Mohammed Benaissa, 2018 Depression Detection From Eye Blink Features

[14] Lang He, Dongmei Jiang, and HichemSahli 2018. Automatic Depression Analysis using Dynamic Facial Appearance Descriptor and Dirichlet Process Fisher Encoding

[15] Hassan Ali Mohammadi Motlagh, Behrouz Minaei Bidgoli, Akbar Parvizi Fard 2017, Design and implementation of a web-based fuzzy expert system for diagnosing depressive disorder.

[16] Anushri Arora, Akanksha Joshi, Kruttika Jain, Shashank Dokania, Dr. Pravin Srinath 2018, Unraveling Depression Using Machine Intelligence

[17] Vishal Kumar Gupta, Pooja Asthana, 2019. Role of Artificial Intelligence in Dealing with Emotional and Behavioural Disorders

[18] Uditesh Jha, Keyur Khant, Milan Kotadiya, Kirti Gamdha, Prof. Zalak Kansagra 2019 To Alleviate Depression by Interactive Artificial Conversation Entity
[19] Hengjin Ke Chen, Tejal Shah,Xianzeng, Liu,Xinhua, Zhang Lei, Zhang Xiaoli Li Cloud-aided online EEG classification system for brain healthcare: A case study of depression evaluation with a lightweight CNN.

[20] AM Rahman, Abdullah Al Mamun, Alma Islam, 2017 Programming challenges of Chatbot: Current and Future Prospective.

[21] Andrea Gaggioli 2019. Online Emotion Recognition Services Are a Hot Trend

[22] Libby Ferland, Ziwei Li, Shridhar Sukhani, Joan Zheng, Luyang Zhao,2018. Maria Gin Assistive AI for Coping with Memory Loss

[23] Emily Harris Canning, Robert D Canning, 2015. Depression Symptoms And Adaptive Style in Children

[24] Darren Foster, Carolyn McGregor, 2017. A survey of Agent-Based Intelligent Decision Support Systems to Support Clinical Management and Research

[25] Borja Martinez-perez, Isabel de la Terre-Diez, 2013. Mobile Health Applications For Most Prevalent Conditions By World Health Organization: Review And Analysis

[26] Will Knight, 2018. Your smartphones Ai algorithms could tell if you are depressed

[27] Anees Ul Hassan, Jamil Hussain, Musarrat Hussain, Muhammad Sadiq, Sungyoung Lee, 2017. Sentiment Analysis of Social Networking Sites (SNS) Data using Machine Learning Approach for the Measurement of Depression

[28] Kaustubh Kulkarni, Ciprian Adrian Corneanu, Ikechukwu Of odile, 2017. Automatic Recognition of Facial Displays of Unfelt Emotions

[29] N Wani, D Bodade, S Gunjal, V Mahadik, 2016. A Survey on: Image Process using Two-Stage Crawler International Journal of Computer Applications 975, 8887

[30] Bharat, V., Shelale, B., Khandelwal, K. and Navsare, S., 2016. A review paper on data mining techniques. International Journal of Engineering Science and Computing (IJESC), 6(5), pp.6268-6271. 